\title{
ESPACIOS DISCURSIVOS: UNA SOLUCIÓN AL PROBLEMA DE LA HETEROGENEIDAD DISCURSIVA
}

\author{
Liana Pop \\ Universidad Babeș - Bolyai (Cluj)
}

El propósito del presente artículo es mostrar las ventajas de una perspectiva de modelación y análisis del discurso en los términos que llamamos en otra parte «espacios discursivos», es decir, capas referenciales, virtual y simultáneamente al alcance de los hablantes, tanto en el proceso de producción, como en el de interpretación del discurso (Pop, 2000).

\section{1. ¿QUÉ SON LOS ESPACIOS DISCURSIVOS?}

Si partimos de la constatación que las rupturas gramaticales funcionan, en el interior de las «frases», como indicadores de unas funciones discursivas de lo más diversas, podemos considerar que el tratamiento de las funciones discursivas (del tipo acto verbal) en los términos de 
macro-tipos de funciones (parecidas a las funciones «de comunicación» de Jakobson, pero que hemos llamado operaciones) podría homogeneizar la visión sobre el discurso. De esta manera, hemos establecido como tipos fundamentales las siguientes operaciones discursivas (representadas globalmente en una tabla con varios niveles, que sugiere la simultaneidad en los procesos de puesta en discurso o de interpretación):

Espacios del discurso:

\begin{tabular}{|c|c|}
\hline Id el espacio interdiscursivo & $\begin{array}{l}\text { operaciones kintertextuales»; reite- } \\
\text { raciones, citas... }\end{array}$ \\
\hline Md el espacio metadiscursivo & $\begin{array}{l}\text { operaciones metadiscursivas: refor- } \\
\text { mulaciones, precisiones, explicacio- } \\
\text { nes, ejemplos }\end{array}$ \\
\hline Ip el espacio interpersonal & $\begin{array}{l}\text { operaciones de llamamiento al inter- } \\
\text { locutor: saludos, nocativoss, cimpe- } \\
\text { rativoss }\end{array}$ \\
\hline s el espacio subjetivo & $\begin{array}{l}\text { operaciones subjetivas: evaluaciones, } \\
\text { puesta de relieve, estados afectivos }\end{array}$ \\
\hline $\begin{array}{l}\text { D el espacio descr, «del } \\
\text { mundos }\end{array}$ & $\begin{array}{l}\text { operaciones descriptivas: constata- } \\
\text { ciones, informaciones, descripciones }\end{array}$ \\
\hline pp el espacio presuposicional & $\begin{array}{l}\text { operaciones acerca del fondo de co- } \\
\text { nocimiento: regresiones, encuadra- } \\
\text { miento, tematizaciones, explicaciones }\end{array}$ \\
\hline Pd el espacio paradiscursivo & $\begin{array}{l}\text { operaciones de formulación: búsque- } \\
\text { da de la «palabra apropiaday, hesita- } \\
\text { ciones, errores... }\end{array}$ \\
\hline Pro el espacio prosódico & $\begin{array}{l}\text { operaciones suprasegmentales: en- } \\
\text { tonación, pausas.. }\end{array}$ \\
\hline Is & $\begin{array}{l}\text { operaciones no-verbales: gesto, mí- } \\
\text { mica, mirada, proxémica.. }\end{array}$ \\
\hline
\end{tabular}

Así, en un fragmento de discurso como es (1):

(1) RICÃ: No grites, madam (se arrastra un paso de rodillas) ¡Sé misericordiosa! Estoy loco de amor; sí....... (Caragiale)

las rupturas gramaticales y, por lo tanto, las relaciones gramaticales cero, están marcadas por varios signos de puntuación; dos puntos, coma, 
paréntesis, exclamación, punto y coma. Se comprueba que, realmente, estos «signos» son aquí indicadores, por lo tanto casos marcados, de unos pasos hacia «otra cosa», cada vez hacia otro tipo de referencia, $o$, dicho de otra manera, hacia una operación diferente del segmento en curso. El resultado global es el de una secuencia de actos extremadamente heterogéneos, compuestos por:

- Un segmento nominal que identifica, en el texto dramático, al personaje/al hablante (Ricã).

- Un segmento oracional (ruego: No grites).

- Un vocativo (llamada: madam).

- Una didascalia, especificando la postura, marcada por paréntesis (se arrastra un paso de rodillas).

- Otro segmento oracional (imploración: ¡Sé misericordiosa!).

- Otro segmento oracional (confesión/declaración de amor: Estoy loco de amor).

— Un segmento adverbial (confirmación: sî).

Esta heterogeneidad se puede representar en el caso de esta secuencia en términos de «espacios discursivos» y numerando los segmentosactos de 1 a 7 , según sigue:

(1') RICÃ: $1 /$ No grites, $2 /$ madam, $3 /($ se arrastra un paso de rodillas) ${ }^{4} /$ iSé misericordiosa! ${ }^{5} / /$ Estoy loco de amor; $6 /{ }^{6},{ }^{7} /$ :

\begin{tabular}{|c|c|c|c|c|c|}
\hline 1 & 2 & 3 & 4 & 5 & 6 \\
\hline Md Rică: & $\begin{array}{l}\text { No } \\
\text { grites, }\end{array}$ & & & & \\
\hline$I p$ & No grites, & madam, & & $\begin{array}{l}\text { Sé } \\
\text { misericordiosa! }\end{array}$ & \\
\hline$s$ & & & & $\begin{array}{l}\text { Se } \\
\text { misericordiosa! }\end{array}$ & $\begin{array}{l}\text { Estoy loco de si,... } \\
\text { amor, }\end{array}$ \\
\hline$D$ & & & & & $\begin{array}{l}\text { Estoy loco de } \\
\text { amor, }\end{array}$ \\
\hline pp Rică: & & & & jsé misericordios & \\
\hline Pro: & , & ? & ) & ! & $\ldots$ \\
\hline Is & & & $\begin{array}{l}\text { (se arrastra un } \\
\text { paso de rodillas) }\end{array}$ & & \\
\hline
\end{tabular}


Resulta (de arriba abajo, en el cuadro) un conjunto de operaciones:

- Metadiscursivas $M d$ ( 1 verbo de decir implícito; 2 verbo explicitando la modalidad del decir: gritar).

- Interpersonales $I p(2,5$ llamamientos implícitos en imperativo; 3 , llamamiento explícito en vocativo).

- Subjetivas $s$ (5, 6 evaluaciones a través de misericordiosa, loco; confirmación $s \hat{i})$.

- Descriptivas $D$ (6, declaración Estoy loco de amor).

- Suposicional $p p$ (1, mencionando a la persona que habla; 2 , justificando el ruego).

- Prosódicas Pro (signos de puntuación como fronteras de acto).

- Intersemióticas Is (no-verbales 4 se arrastra un paso de rodillas).

Se observa que los indicadores de transición de un tipo de operación a otra son, cada vez, para esta secuencia escrita:

- Distintos signos de puntuación, representados en nuestro cuadro en el espacio destinado a las operaciones prosódicas Pro (en orden:,,() $i ; \ldots)$.

- Pero también otros recursos del código grafico: MAYÚSCULAS para indicar al personaje que habla; itálicas para las didascalias.

Está claro que la representación del texto (aquí, texto dramático) moviliza varios indicadores, más o menos convencionales, para facilitar la lectura/interpretación de las heterogeneidades del discurso.

Trataré de mostrar a continuación las ventajas de este tipo de modelización del discurso, visto como múltiples virtualidades de «espacios de maniobra».

\section{La posibilidad de una integración gramática-discurso}

En la secuencia anterior hay 4 segmentos «inanalizables» gramaticalmente: 
- El segmento nominal Ricã aislado por dos puntos (1).

- El vocativo madam, aislado por el par de comas (3).

- La didascalia incidente se arrastra un paso de rodillas, aislada por el par de paréntesis (4).

— «El adverbio» pro-frase sí, aislado entre dos comas (7).

Si bien estos segmentos llamados «inanalizables» no se incluyen en los tipos de relaciones gramaticales canónicas, no significa que la secuencia en causa no sea perfectamente aceptable desde el punto de vista comunicativo, al contrario: semejantes «frases» se producen y se interpretan sin ningún problema por todos los hablantes. Sólo que el análisis de tipo gramatical es incapaz, con los instrumentos a su alcance, de dar cuenta de la totalidad de las relaciones que se compromete en las dos «frases» ${ }^{1}$ de la réplica dramática arriba mencionada. Ya que, además de las relaciones gramaticales, actualizan relaciones de tipo discursivo, e incluso general comunicativas (entre actos verbales y no-verbales), suponiendo, por consiguiente, no sólo una configuración de «lugares gramaticales», sino también de «lugares discursivos».

Además de las relaciones gramaticales canónicas (véase cada segmento-acto de 1 a 7), considero que lo que tiene la capacidad de efectuar la representación que propongo es precisamente la integración, en este cuadro de virtualidades discursivas, de todos los segmentos que se encuentran en relación gramatical cero (por lo tanto excluidos de las relaciones lícitas de la gramática), y que los integra como segmentos situados en una relación discursiva... En otras palabras, esta representación es, por lo tanto, capaz de conferir espacios virtuales a estos tipos no-gramaticales de relaciones, y de indicar pasos, de tipo discursivo, entre

- 1 y 2-7: estamos ante una inferencia de tipo metadiscursivo (a través de Ricã dijo), en la que (1) ocupa simultáneamente los espacios metadiscursivos $M d$ y supuestamente $p p$, como acto constituido por operaciones de este tipo.

${ }^{1}$ Digo «frases» entre comillas, porque, a pesar de la apariencia de frases propiamente dichas, la secuencia en causa no está formada por frases en el sentido gramatical, sino solamente en el discursivo (Roulet, 1994). 
- 2 y 3: un acto de orden y un apelativo (la persona a la que se dirige); (2), el acto de orden No grites, está compuesto de una operación de tipo interpersonal Ip y una de tipo metadiscursivo $M d$ (el verbo gritar); (3), el acto de apelación madam, está constituido de una operación única de apelación del interlocutor $I p$.

- 3 y 4: ninguna relación directa, no-mediada; (4), acto no-verbal de arrastrarse de rodillas, está representado como operación noverbal, en el espacio llamado intersemiótico Is.

- 5 y 2: una relación explicativo-justificativa para el acto (2) (operación suposicional $p p$ ).

- 4 y 5: relación de modalidad enunciativa (gesto acompañando la súplica), muy probable en realización simultánea; la súplica (5) ¡Sé misericordiosa! es aquí un acto compuesto de una operación de dirigirse $I p$, una de evaluación subjetiva $s$ y una suposicional, de justificación del ruego anterior $p p(2)$.

- 6 y 7: relación de confirmación; el acto declarativo Estoy loco de amor (6) ocupa el espacio descriptivo $D$ y el subjetivo $s$; el acto (7) es una operación subjetiva $s$.

En cuanto a la ruptura entre 3 y 4 , la representación en términos de espacios discursivos es integrada naturalmente como perteneciendo a las posibles operaciones comunicativas, que admiten que un acto noverbal (el arrodillarse) coexista, incluso sin que sea obligatorio, como en este caso, con el tipo de acto verbal de implorar ${ }^{2}$.

Según vemos, el cuadro de representación de las virtualidades discursivas puede funcionar como principio de cohesión para los segmentos no-integrados gramaticalmente. «Las rupturas» gramaticales se re-interpretan fácilmente, desde esta perspectiva, como relaciones discursivo-comunicativas, y los lugares/papeles gramaticales se alternan o incluso se sobreponen a los discursivos.

Este último caso puede ser ilustrado por el de las posiciones dobles, ocupadas por un elemento o segmento que tiene una función difusa, gramatical y discursiva al mismo tiempo, hecho indicado a través del tipo de notación. Los adjetivos dislocados son un ejemplo de este tipo,

${ }^{2} \mathrm{El}$ acto de implorar parece definirse como un ruego potenciado por marcas suplementarias-verbales (te suplico) o no-verbalès (juntar las manos, arrodillarse, etc.) 
al igual que los relativos explicativos, etc., de hecho lo son todas las «funciones supuestamente "explicativas" (vs. «determinativas») de las gramáticas clásicas. Operan no sólo «transiciones» de tipo discursivo (v. los diferentes espacios ocupados por el adjetivo «explicativo» 2 - en el ejemplo 2 -, respectivamente por «los relativos explicativos» 3 y 8 - en el ejemplo 3-: en ambos casos estamos ante actos llamados en general explicaciones, dislocadas con marcas forte, como son las comas o los guiones. Además de esta notación que indica operaciones/actos discursivos distintos del enunciado-base, los segmentos en causa guardan sin embargo también una notación gramatical, el acuerdo en género y número del adjetivo leída (en 2), acuerdo y nexo gramatical respectivamente de quien (en 3):

(2) Mi suerte es haber tenido una madre, ${ }^{1} /$ leída realmente $2 /$ una persona que, ${ }^{3}$ '/por lo menos la mitad de su vida, ${ }^{4} /$ ha leído. ${ }^{3}$ /(Ileana Vulpescu):

\begin{tabular}{|c|c|c|c|c|c|}
\hline 8 & 1 & 2 & 3 & 4 & $3^{\prime \prime}$ \\
\hline$M d$ & & & $\begin{array}{l}\text { ma persona } \\
\text { que. }\end{array}$ & & ha \\
\hline s & $\begin{array}{l}\text { Mi suerte ... una } \\
\text { madre, }\end{array}$ & lefa realmente, & & $\begin{array}{l}\text { por lo menos la } \\
\text { mitad de su vida, }\end{array}$ & \\
\hline$D$ & $\begin{array}{l}\text { Mi suerte . tha } \\
\text { madre. }\end{array}$ & Terda & $\begin{array}{l}\text { una persona } \\
\text { que, }\end{array}$ & & $\begin{array}{l}\text { ha } \\
\text { lefdo }\end{array}$ \\
\hline$p p$ & & & $\begin{array}{l}\text { una persoma } \\
\text { que, }\end{array}$ & $\begin{array}{l}\text { por lo menos la } \\
\text { mitad de su vida, }\end{array}$ & $\begin{array}{l}\text { ha } \\
\text { leído. }\end{array}$ \\
\hline
\end{tabular}

(3) En la editorial, $1 /$ en el momento en el que se supo que Ruth ${ }^{2} /$ a quien hasta entonces la llamaban en un tono de alta deferencia «camarada Volintiru» [...] $3 /$ - cuando se supo que $4 /$ no iba a volver, ${ }^{4} /$ de repente, ${ }^{5} /$ Ruth se convirtió en, ${ }^{6}$ '/a regañadientes y en total confianza, ${ }^{7 /}$ «perro judío», ${ }^{11} /$ a quien se le aplicaban todas las injurias guardadas hasta entonces; ${ }^{8} /($ Ileana Vulpescu).

\begin{tabular}{|l|c|c|c|c|c|c|c|c|c|c|}
\hline$l d$ & & & $-3-$ & & & & & & "6" " & $8 ;$ \\
\hline$M d$ & & & $-3-$ & & & & $6 \%$ & & & $8 ;$ \\
\hline$S$ & & & $-3-$ & & & 5, & & 7, & & \\
\hline$D$ & 1, & 2 & & & 2, & & 6, & & & \\
\hline$P d$ & & & & 4 & & & & & & \\
\hline$p p$ & 1, & 2 & $-3-$ & & & & & & & \\
\hline
\end{tabular}


La dislocación aparece, en esta visión, como marca de derivación ilocucional, o lo que llamamos conversión pragmática, transformando una función gramatical en función discursiva:

- El sujeto o el complemento dislocado se convierte en tema (v. escarlatina y tifus complementos directos tematizados en 4 , más abajo).

- El adjetivo-explicación/evaluación/invectiva ( 2 en $2 ; 6$ " en 3 ).

- La relativa-explicación (3, 8 en 3).

- El circunstancial de lugar y/o tiempo-marco (4 en 2; 1, 2 en 3).

- El circunstancial de causa-justificación.

— El adverbial-comentario (5,7 en 3) etc. (Pop, 2000).

(4) Verán. ${ }^{1}$ /Escarlatina tuve hace cuatro años, ${ }^{2} /$ tifus,${ }^{3} /$ en la elección parcial de hace año y medio. ${ }^{4} /$ (Baranga):

\begin{tabular}{|l|c|c|c|c|c|}
\hline & 1 & & 2 & 3 & \\
\hline$I p$ & Verán & & & & \\
\hline$S$ & & & tuve hace cuatro años, & & \\
\hline$D$ & & Escarlatina & tuve hace cuatro años, & tifus, & $\begin{array}{l}\text { en la elección parcial de hace } \\
\text { af́o y medio. }\end{array}$ \\
\hline$p p$ & & Escarlatina & & tifus, & \\
\hline
\end{tabular}

Si la dislocación como procedimiento de tematización no está marcada más que desde el punto de vista de la posición (ante-posición) y diferenciación de espacios en el segmento 2 escarlatina, en cambio en el segmento 3 tifus, se completa con una marca prosódica (la coma, como frontera de acto). La representación con la ayuda de los espacios discursivos puede dar cuenta de estos matices, que corresponden en realidad a la descripción de unos grados de tematización diferentes (como simple operación o como acto propiamente dicho de tematización; y v. más adelante el párrafo 5).

\section{La posibilidad de la integración léxico-discurso}

Según podemos observar en el ejemplo (2), algunas relaciones discursivas toman como referencia el ámbito mismo del discurso y van a 
ser consideradas de esta manera relaciones metadiscursivas. Las que, precisamente, toman su referencia en el ámbito léxico pueden actualizar las relaciones léxico-semánticas como relaciones de tipo discursivo. Así funcionan las cosas, por ejemplo, con los segmentos 2 (una madre) leída y 3 una persona que ha leído del ejemplo (2) arriba mencionado, en el cual una definición léxica («leída» = «persona que ha leído») se actualiza como relación discursiva (en el espacio metadiscursivo $M d$, presuposicional $p p$ - una definición es generalmente conocida -y descriptivo $D$ - una definición, en general, describe).

Hay también otras relaciones al nivel del sistema léxico que se pueden actualizar como posiciones discursivas, y se puede decir desde este punto de vista que representan virtuales operaciones/actos al nivel del discurso. La sinonimia, por ejemplo, que, en sucesión, se transforma en precisión, va a ocupar con prioridad todo el espacio metadiscursivo $M d$, como en el ejemplo (5) abajo mencionado, donde la palabra 1 «el preludio» va a ser precisada/reformulada a través de 4 el comienzo:

(5) Las elecciones que van a tener lugar en la capital de nuestra localidad, ${ }^{1}$ /es decir, ${ }^{2} / ¿$ verdad?, ${ }^{3} / \mathrm{de}$ ustedes, ${ }^{2}$ /son sólo el preludio, ${ }^{1 "} /$ sólo el comienzo $4 /$ del grave enfrentamiento político que se desarrollará en todo el territorio del país ${ }^{1 '}$ "/(Baranga):

\begin{tabular}{|l|r|c|c|c|c|c|c|}
\hline & 1 & 2 & 3 & $2 "$ & $1 "$ & 4 & $1 "$ \\
\hline$M d$ & & es decir & & & & $\begin{array}{l}\text { sólo el } \\
\text { comienzo }\end{array}$ & \\
\hline$I P$ & localidad, & & verdad, & de ustedes, & & & \\
\hline$s$ & localidad, & & & & & $\begin{array}{l}\text { sólo el } \\
\text { comienzo }\end{array}$ & del grave.. \\
\hline$P$ & Las elecciones que... & & & & $\begin{array}{l}\text { son sólo } \\
\text { el preludio }\end{array}$ & & del grave... \\
\hline Pd & localidad & & verdad, & & $\begin{array}{l}\text { son sólo } \\
\text { el preludio }\end{array}$ & & \\
\hline Pro & & &, & &, & & \\
\hline
\end{tabular}

Las series sinonímicas favorecen la aparición de cadenas de operaciones meta-discursivas, con reformulaciones o reajustes en el discurso, como es el caso del ejemplo (6):

(6) Ustedes representan, $1 /$ iverdad?, $2 /$ los capos de nuestra organización en la ciudad, $1 " / y$ si no me equivoco, $3 /$ en la provin- 
cia, ${ }^{1 " \prime} /$ Como se diría, $4 /$ la gente importante. ${ }^{5 /(D e l e t r e a n d o) .}{ }^{6 / L a s}$ personalidades, ${ }^{7} /$ nuestra gente de confianza ${ }^{8} /(\mathrm{Baranga})$ :

\begin{tabular}{|l|c|c|c|c|c|c|c|c|c|}
\hline$I d$ & & & & & & 4 & & & \\
\hline$M d$ & & & & & & & & & 8 \\
\hline$M d$ & & & & & & & & 7 & \\
\hline$M d$ & & & & & & & 5 & & \\
\hline$M d$ & & & $1^{\prime \prime}$ & & & 4 & & & \\
\hline$I p$ & 1 & 2 & & & & & & & 8 \\
\hline$s$ & & & $1^{\prime \prime}$ & 3 & & & & & 8 \\
\hline$D$ & $1^{\prime}$ & & $1^{\prime \prime}$ & & $1^{\prime \prime \prime}$ & & & & \\
\hline Pd & & 2 & & 3 & & & & & \\
\hline Pro & & & & & & & & 6 & 6 \\
\hline
\end{tabular}

Otras relaciones semánticas del sistema léxico son también virtuales operaciones/actos discursivos:

- La metáfora-operación virtual de reformulación/reclasificación (v. 1 " en 6).

- La paronimia-operación virtual de formulación $(P d)$.

- Antonimia-operación virtual de contradicción $(s)$.

\section{Linealidad vs. densidad del discurso}

La segmentación de la cadena discursiva en capas/«espacios» da cuenta, como hemos visto, de la composición de los actos verbales en el discurso. Como estos espacios representan en general los ingredientes de la comunicación en esta representación con muchas capas, un acto verbal aparece compuesto por una sola o varias operaciones discursivas fundamentales. Véase, por ejemplo:

- El acto 5 de declaración de amor en (1), ocupando los espacios descriptivo $D$ y subjetivo $s$.

- El acto 1" de calificación metafórica en (6), con una configuración de operaciones, descriptiva $D$, subjetiva $s$ y meta-discursiva $M d$. 
- El acto 8 de reformulación en (6), una configuración compleja de operaciones, subjetiva $s$, interpersonal $I p$ y meta-discursiva $M d$.

La visión lineal del discurso puede ser desmitificada de esta manera, y las relaciones gramaticales, consideradas de costumbre homogéneas, se completan necesariamente con relaciones de tipo discursivo, vistas, en conjunto, como transiciones más o menos abruptas entre distintas configuraciones de operaciones en el discurso. La linealidad estricta va a poder ser sustituida entonces, por un lado, por una sucesión más permisiva, y por otro, por una consistencia semántico-pragmática diversificada.

\section{La descripción del discurso como cuadro de virtualidades}

La representación del discurso como superposición de espacios de maniobra, que los hablantes gestionan simultáneamente, ocupando al mismo tiempo o sucesivamente un espacio u otro/una configuración de espacios u otra, parece tener la ventaja de dar cuenta, globalmente, no sólo de sus virtualidades excesivas (verbal, para-verbal y no-verbal), sino también de sus virtualidades semántico-pragmáticas, de la totalidad de las operaciones discursivas susceptibles de producirse en el discurso. Aunque el inventario de «espacios» es (y probablemente lo sea siempre) relativo, describir el discurso de este modo, aunque todavía bastante rígido, me parece más cercano a sus representaciones intuitivas (como, por ejemplo, la metáfora corriente del discurso como flujo, correr, en el que inevitablemente se suponen mezcladas varias «aguas», vías...). Esta manera de abordar deja de ser obligatoria y obsesivamente jerárquica, tomando en cuenta, en primer lugar, las heterogeneidades de toda clase, necesariamente de manera jerárquica. Una integración de todos los tipos de operaciones discursivo-comunicativas se vuelve posible de esta manera:

- Operaciones expresivas segmento-verbales, en las que semánticamente se puede dec̀idir el tipo de operación general-comunicativa que implica un segmento: los verbos del decir -operaciones metadiscursivas e inter-discursivas (v., por ejemplo, 3 a quien hasta 
entonces la llamaban... en 3; 4 como se diría en 5); las palabras axiológicas - operaciones subjetivas de tipo evaluación, calificación (v., por ejemplo la declaración 5 en 1; las calificaciones 1 y 8 en 5) etc.

- Operaciones expresivas para-verbales: la búsqueda de la expresión, de la palabra adecuada, errores expresivos. En el ejemplo (7) abajo mencionado, los segmentos de búsqueda de la expresión y los errores de expresión, eliminados de los análisis jerárquicos en general por parásitos, están representados aquí en el espacio de maniobra que el hablante tiene a mano para todas las operaciones de (pre) formulación en general:

(7) Ha salido la gente fuera $1 /$ estaba abarrotado $2 / n i^{3} / \mathrm{se}$ iba nadie que si eramos pocos nos mataba a todos, $4 /$ pero la gente gritaba no nos vamos $5 / \ll$ no nos vamos de aquí, no nos vamos a casa» $6 /$ no nos vamos porque si somos muchos venceremos $7 / y$ si aquel bandido de $8 /$ aquél $9 /$ cómo se llamaba $10 /$ que era primer ministro $11 /$ era aquel ${ }^{12} /$ Dăscălescu 13/estaba allí 14 /injuriaba en el micrófono allí 15 /éstos entonces «no me insultes ${ }^{16} /$ tío ${ }^{17}$ /que entramos y te sacamos los ojos tío» ${ }^{18} /$ (corpus oral Pop):

\begin{tabular}{|c|c|c|c|c|c|c|c|}
\hline$M d$ & 8 & 9 & 10 & 11 & 12 & 13 & 14 \\
\hline Id & & & $\begin{array}{l}\text { cóno se } \\
\text { llamaba }\end{array}$ & & & Dăscălescu & \\
\hline$M d$ & & & $\begin{array}{l}\text { cómo se } \\
\text { lamaba }\end{array}$ & & & Dăscălescu & \\
\hline$I p$ & aquel & aquet & $\begin{array}{l}\text { cómo se } \\
\text { llamaba }\end{array}$ & & & & \\
\hline$s$ & bandido & & & & & & \\
\hline $\bar{D}$ & $\begin{array}{l}\text { y si aquel } \\
\text { bandido de }\end{array}$ & & & & & & \\
\hline$p p$ & & & & $\begin{array}{l}\text { que era primer } \\
\text { ministro }\end{array}$ & era aquel & & estaba allí \\
\hline$P d$ & & aqué & $\begin{array}{l}\text { cómo se } \\
\text { llamaba }\end{array}$ & & era aquel & & \\
\hline Pro & - & - & - & - & - & 1 & 1 \\
\hline
\end{tabular}

- Operaciones expresivas no-segmentadas (prosódicas): signos de puntuación, pausas, contornos de entonación, maneras de decir 
etc. En el ejemplo (7) de más arriba, los contornos «suspendidos» de búsqueda de la expresión son generalmente estables (-), los de la cima ascendentes $(/)$, y los conclusivos descendentes () . En el ejemplo (6), el segmento 6 (deletreando), mencionando el modo de decir de los segmentos 7 y 8, se sobreimprime a éstos en el espacio reservado a la prosodia Pro. En todo caso, los signos de puntuación y prosódicos funcionan discursivamente como «indicadores de transición de nivel», marcando cada vez la transferencia hacia otra operación discursiva o hacia otra configuración de operaciones.

- Operaciones expresivas no-verbales: gestos, mímica. Hemos observado el modo en el que lo no-verbal (didascalia 6 en el ejemplo 1) integra naturalmente lo verbal. Si en el código gráfico la convención pide el encadenamiento de los segmentos en estricta linealidad, en términos de espacios discursivos lo no-verbal se puede representar como sobre-impreso a lo verbal o no.

\section{La posibilidad de distinguir entre acto y operación discursiva}

Presentamos abajo dos secuencias (8) y (9) en las que hemos representado, normalmente, uno de los inanalizables gramaticales, una interjección, en diferencia de espacios con la secuencia-base:

(8) $\mathrm{Di},{ }^{1} /$ por Dios, ${ }^{2} /$ ¿qué es lo que no te va? ${ }^{3}$

\begin{tabular}{|l|c|c|c|}
\hline Ip & Di, & por Dios, & qué es lo que no te va? \\
\hline$M d$ & & por Dios, & \\
\hline$s$ & & por Dios, & \\
\hline Pro &, &, & $?$ \\
\hline
\end{tabular}

(9) En cuanto a quien no se conforma con mi manera de escribir [el cuento], le ruego, si es un maestro, que escriba uno que le haga famoso, que por Dios no me enfado si es bonito y bien escrito (Dragoslav):

\begin{tabular}{|l|l|r|r|}
\hline$M d$ & & por Dios & \\
\hline$s$ & que & por Dios & no me enfado... \\
\hline
\end{tabular}


Se observa que la misma interjección (una «palabra-frase», que forma de hecho los actos de juramento (en 9) o de solicitud de juramento (en 8), se presenta cada vez con diferencia de espacios frente al enunciado-base, pero que a nivel prosódico, los indicadores de integración en el enunciado difieren:

- En la primera ocurrencia, por Dios aparece como acto marcado (véase el par de comas, específico a los incidentes, funcionando, de hecho, como fronteras de acto, señal de no-integración prosódica en el enunciado-base).

- Mientras que en la segunda ocurrencia, por Dios aparece sólo como operación discursiva, integrada prosódicamente en el enunciado-base «que no me enfado si es bonito y bien escrito».

La representación en capas o «espacios» de las diferencias entre operaciones (diferencias semántico-pragmáticas) se completa, en este tipo de modelación, con la representación prosódica de las fronteras de acto: en el caso en que la frontera está marcada, diré que estamos ante una operación fuerte, emergente, y en el caso en el que la frontera no esté marcada, con una operación de fondo, más débil. Considero, en términos cognitivistas:

- Que una operación doblemente marcada representa una figura discursiva fuerte, lo que intuitivamente se llama un acto y recibe denominaciones «populares» en todos los idiomas.

- Y que una operación de fondo puede ser como mucho considerada, en los mismos términos cognitivistas, un semi-acto. Para esta última, marcada con más ligereza, sólo a través de lo que se puede llamar huellas discursivas, prefiero mantener la denominación de operación pura y simplemente.

En el tipo de representación que propongo, las operaciones no aparecen, por lo tanto, más que en diferencia simple, semántica (entre espacios), mientras que los actos aparecen en doble diferencia, tanto semántica (entre espacios), como prosódica (segmentación con fronteras de acto, verticales). Frente a los actos-unidades, las operacio- 
nes aparecerán como menos-que-unidades: modalizadores, marcas, conectores, adverbios de enunciación, regularizadores, partículas, «indicios», errores, «puntuaciones», etc. (Pop, 2000a, b).

\section{Los no-actos}

La perspectiva sobre lo que no se clasifica de costumbre como acto (en general a causa del contenido oracional explícito o de la naturaleza más «obscura» de la operación que cumple el segmento) se puede homogeneizar si consideramos que todo es operación discursiva y que, tal y como se ha visto, no todas las operaciones se actualizan como actos. Las que tienen un grado bajo de predicatividad preferirán normalmente el estatuto de no-acto, y bajo esta categoría muy amplia se van a incluir en general las operaciones no-descriptivas, y de éstas más bien:

- Las subjetivas (los adverbios de enunciación, los modalizadores, más o menos integrados en la «frase»).

- Las meta-discursivas, reformulaciones, que puntualizan.

- Las para-discursivas: errores, palabras incidentes.

- Las interpersonales: regularizadores, apelaciones, etc.

Al igual que en el caso de las interjecciones (v. por Dios en los análisis de más arriba, 8 y 9), se puede remarcar el hecho de que el estatuto de estos segmentos oscila entre el de acto o no-acto. En el caso de unas fórmulas oracionales, la decisión se toma con más facilidad y el estatuto de acto, a pesar de la reducción a una «fórmula», es plausible. En el caso de los segmentos no-oracionales, llamados «predicativos» o no, las fronteras de acto representan un tipo de marca con cierta dificultad en la decisión de la categoría. De tal modo que, en el ejemplo (7) arriba mencionado, aquél para-discursivo al nivel del segmento 9 puede ser más fácilmente considerado acto (y se delimita en consecuencia) por el hecho de que está marcado prosódicamente como «isla»; en cambio, el mismo aquel para-discursivo del segmento 12 , no-delimitado el mismo por fronteras de acto, no va a poder ser considerado más que una huella 
de una operación de formulación, integrándose en un enunciado más amplio. En todo caso, en cualquier perspectiva de análisis de tipo jerárquico, este «error» expresivo no se habría tomado en cuenta.

Lo mismo pasa con las dos ocurrencias del apelativo tío de (6'):

(7') entonces éstos «no me insultes $16 / t i o{ }^{17}$ /que entramos y te sacamos los ojos tío» ${ }^{18} /$ (corpus oral Pop), donde la primera, 17, está aislada desde un punto de vista prosódico, por lo tanto hace figura de acto (de amenaza), en cambio la segunda, integrada en 18, más tenue, menos emergente, se quiere sólo una huella del mismo tipo de operación, y es interpretable, más bien, como modalizador subjetivo de desprecio.

Los espacios movilizados por esta palabra serían, para ambas ocurrencias, pero con ponderación diferente: $I p$ (operación de apelación), $s$ (actitud subjetiva).

\section{La posibilidad de seguir «el hilo del discurso»}

La representación del discurso a través de «espacios» puede finalmente dar cuenta de lo que normalmente se llama «el hilo del discurso», como también de las situaciones en las que los hablantes «lo pierden». Al ser las digresiones nada más ni nada menos que la imbricación del discurso en otro espacio que el pensado inicialmente como «principal», presento a continuación la visualización de este fenómeno al nivel de los «espacios».

En el texto ya analizado arriba (3), vamos a destacar esta vez los espacios que cobijan las operaciones principales en un momento o en otro del desarrollo del discurso:

(3') En la editorial, $1 /$ en el momento en el que se supo que Ruth ${ }^{2} /$ - a quien hasta entonces la llamaban en un tono de alta deferencia «camarada Volintiru» y no se olvidaban de añadir para las personas que no la conocían, «saben, la esposa del camarada Volintiru», señalando hacia arriba con la mano y con la cabeza, para indicar la posición del «camarada Volintiru» $3 /$ - cuando se supo que $4 /$ no iba a volver, $2 " / \mathrm{de}$ repente, ${ }^{5} /$ Ruth se convirtió en, ${ }^{6} / \mathrm{a}$ regañadientes y en total confian$\mathrm{za}, 7 /$ «perro judío», 6"/a quien se le aplicaban todas las injurias guardadas hasta entonces; $8 /$ (Ileana Vulpescu): 
ESPACIOS DISCURSIVOS: UNA SOLUCIÓN AL PROBLEMA DE LA HETEROGENEIDAD...

\begin{tabular}{|l|c|c|c|c|c|c|c|c|c|c|}
\hline$I d$ & & & $-3-$ & & & & & & "6", & $8 ;$ \\
\hline$M d$ & & & $-3-$ & & & & 6, & 7, & & $8 ;$ \\
\hline$s$ & & & $-3-$ & & & 5, & & 7, & & \\
\hline$D$ & 1, & 2 & & & $2 "$, & & 6, & & "6", & \\
\hline$P d$ & & & & 4 & & & & & & \\
\hline$p p$ & 1, & 2 & $-3-$ & & & & & & & \\
\hline
\end{tabular}

Una digresión más importante es el segmento 3, otras más reducidas, 7. Se retoma el hilo retomando efectivamente la expresión «abandonada»/interrumpida (cuando se supo que, en 2' y respectivamente, 4). La representación a través de espacios discursivos puede visualizar, como se ha dicho, el trayecto de la información principal, visto como «hilo conductor», pero hace más perceptibles también otros «hilos»/trayectos discursivos.

En otro fragmento de discurso oral (10):

(10) nosotros nos fuimos $1 /$ huimos ${ }^{2} /$ esta casa no estaba terminada ${ }^{3} / \mathrm{se}$ ha quedado vacía $4 / /$ mi hermano ya se llevó a mamita $5 /$ que yo no podía $6 /$ no estaba en condiciones $7 /$ vivía en una choza $8 /$ en las afueras de Malu'Rosu ${ }^{9} /$ huyendo $10 /$ sin leña ${ }^{11} / \mathrm{de}$ pena $12 / /$ y se la llevó mi hermano $13 /$ alguien le dio una vivienda en un piso ${ }^{14} / \mathrm{en}_{\text {un estudio }}{ }^{15} /$ y se llevó a mamita $16 / /$ y aquí estaba todo vacio ${ }^{17}$ /había dos perros que aullaban 18/no pudimos llevar$\operatorname{los}^{19} /($ corpus oral Pop, según «El memorial del dolor»):

\begin{tabular}{|l|l|l|l|l|l|l|l|l|l|l|l|l|l|l|l|l|l|l|l|}
\hline$M d$ & & & & & & & & 8 & 9 & & & & & & & & & & \\
\hline$M d$ & & 2 & & & & & 7 & & & & & & & & 15 & & & 18 & \\
\hline$I p$ & & & & & & & & & & & & & & & & & & & \\
\hline$s$ & 1 & 2 & & 4 & 5 & 6 & 7 & 8 & & 10 & 11 & 12 & 13 & & & 16 & 17 & & 20 \\
\hline$D$ & 1 & 2 & & 4 & & & & & & & & & & & & & 17 & & \\
\hline$p p$ & & & 3 & & 5 & & & & & & & & 13 & & & 16 & & & 20 \\
\hline$p p$ & & & & & & 6 & 7 & 8 & 9 & 10 & 11 & & & 14 & 15 & & & & \\
\hline$P d$ & & & & & & & & & & & & & 13 & & & 16 & 17 & & \\
\hline
\end{tabular}

El hilo del cuento en (10) es el indicado por cursivas en el texto y grises en el cuadro, mientras que el resto de la secuencia representa digresiones de fondo, supuestamente subjetivas $p p+s$ en su mayoría. Es interesante ver la vuelta de las digresiones retomando los segmentosfrontera a través de paráfrasis (cursivas y negritas en el texto), el fenómeno indicando, al parecer, el cuidado de los hablantes por la coheren- 
cia. Un caso interesante para el estudio sería desde esta perspectiva el funcionamiento de la marca por lo tanto, como es la del texto (11), que indica el comienzo de una digresión y se incluiría en el presente caso en espacios de tipo presuposicional $p p$ de los regresos al segundo plano (o de recuperación de las informaciones de fondo). Se puede ver en la representación de abajo que el cuento propiamente dicho 1 [...] 8: pero [...] tenía como marido a un policía es interrumpido por una incursión en informaciones anteriores, de tipo fondo, marcadas también al nivel de los tiempos verbales por el imperfecto, el pluscuamperfecto, pero en primer lugar por la oración introductiva 2 después me enteré, que justifica prácticamente el regreso en el tiempo.

(11) ... pero $1 /$ después me enteré $2 /$ por lo tanto yo tenía cuarenta años $3 /$ ella me llevaba dos años $4 /$ con cuarenta y pico de años ella ${ }^{5} /$ no había tenido $6 /$ no había estado casada más que una sola vez ${ }^{5} /$ un año y pico $^{7} /$ tenía como marido a un policía ${ }^{8} /$ (corpus oral Pop).

\begin{tabular}{|c|c|c|c|c|c|c|c|c|}
\hline$M d$ & & & & $\begin{array}{l}\text { con cuarenta } \\
\text { y pico .... }\end{array}$ & & & & \\
\hline$s$ & $\begin{array}{l}\text { pero después me } \\
\text { enteré }\end{array}$ & 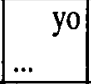 & & & & & & \\
\hline$D$ & & & & & & & & $\begin{array}{l}\text { tenía como marido } \\
\text { a un policía }\end{array}$ \\
\hline$p p$ & $\begin{array}{l}\text { después me } \\
\text { enteré }\end{array}$ & $\begin{array}{l}\text { por lo } \\
\text { tanto } \\
\text { yo... } \\
\end{array}$ & & $\begin{array}{l}\text { con cuarenta } \\
\text { y pico .... }\end{array}$ & & \begin{tabular}{|l|} 
no había \\
estado...
\end{tabular} & & \\
\hline$p p$ & & & $\begin{array}{l}\text { era más } \\
\text { joven... }\end{array}$ & & & & $\begin{array}{l}\text { un año } \\
\text { y pico }\end{array}$ & \\
\hline$P d$ & & & & & $\begin{array}{l}\text { no había } \\
\text { tenido }\end{array}$ & & & \\
\hline
\end{tabular}

Sin duda, la indicación del hilo conductor del discurso, correspondiente a la información con la intención de ser tomada por principal, está probablemente también en el origen de los procesos de selección de la información y de resumen intuitivo.

\section{La tipología secuencial}

En fin, «el cuadro con espacios» no es irrelevante a la hora de indicar distintos tipos de secuencias: 
ESPACIOS DISCURSIVOS: UNA SOLUCIÓN AL PROBLEMA DE LA HETEROGENEIDAD...

- Descriptivo-narrativas (representadas sobre todo en el espacio $D$ ).

- Comentarios subjetivos.

- Comentarios meta-discursivos.

- Cuadros.

- Secuencias dirigidas/dialogadas, etc.

El espacio predominante ocupado por la secuencia va a decidir el tipo al que ésta pertenece. Todo esto se puede ver en las distintas configuraciones de espacios de los análisis arriba mencionados.

Para concluir, pienso, por lo tanto, que la hipótesis de los espacios discursivos que propongo parece tener un poder explicativo y descriptivo apreciable para numerosos aspectos, algunos difíciles, del análisis del discurso.

\section{Referencias bibliográficas}

PoP, Liana (2000a). Espaces discursifs. Pour une représentation des hétérogénéités discursives. BIG 42. Paris-Louvain: Peeters.

- (2000b). «Actes vs. opérations. Pour de nouveaux outils dans l'analyse du dialogue». Actes du Colloque Dialogue Analysis 2000. Bologna, junio 2000 (en prensa).

RoulET, E. (1994). «La phrase: unité de langue ou unité de discours?». En Mélanges de philologie et de littérature médiévales offerts à Michel Burger, Publications romanes et françaises, CCVIII. Droz. 